\section{Cough and dyspnoea is not always bronchial asthma}

\section{Case history}

A 39-yrold female patient who never smoked suffered from chronic dry cough and progressive exercise-induced dyspnoea over a period of 18 months. In 2007, lung volumes were normal, but there was moderate bronchial hyperreactivity as assessed by bronchoprovocation with methacholine. There was a drop in forced expiratory volume in $1 \mathrm{~s}(\mathrm{FEV} 1)$ of $22 \%$ predicted following administration of $0.2 \mathrm{mg}$ methacholine. A diagnosis of asthma was made and the patient was treated with inhaled budesonide combined with formoterol. 1 yr later the patient suffered from an exacerbation of symptoms and was prescribed oral corticosteroids for 2 weeks. A chest radiograph did not show any pathological abnormalities. There was no recovery and the patient was admitted to our outpatient clinic. Physical examination revealed inspiratory rhonchi on the right side.

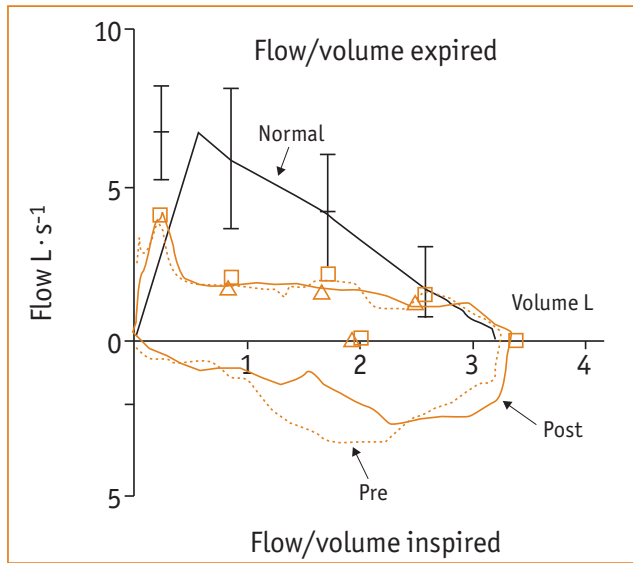

Figure 1

Flow-volume loop, pre- and post-bronchodilator.

Task 1

Interpret the pulmonary function test on the basis of the flow-volume loop (figure 1).
L. Junker ${ }^{1}$

S. Lamm ${ }^{2}$

M. Tamm ${ }^{1}$

D. Lardinois $^{2}$

Clinics of ${ }^{1}$ Pulmonary Medicine and Respiratory Cell Research and ${ }^{2}$ Thoracic Surgery,

University Hospital Basel, Basel, Switzerland

\section{Correspondence}

L. Junker

Clinic of Pulmonary Medicine and Respiratory Cell Research University Hospital of Basel

Petersgraben 4

4031 Basel

Switzerland

junkerl@uhbs.ch

\section{Provenance}

Submitted article, peer reviewed.

Competing interests None declared. 


\section{Answer 1}

The pulmonary function test shows a moderate obstructive ventilatory defect with a forced vital capacity of $3.43 \mathrm{~L}(102 \%$ pred) and FEV 1 of $1.97 \mathrm{~L}(69 \%$ pred) without improvement following bronchodilatation. The flow-volume loop shows flattening of the inspiratory and expiratory curves.

\section{Task 2}

\section{What would be your diagnosis?}

a) Chronic obstructive pulmonary disease

b) Bronchial asthma

c) Bronchial obstruction

d) Advanced pulmonary fibrosis

\section{Task 3 \\ Interpret the computed tomography (CT) section (figure 2 ).}
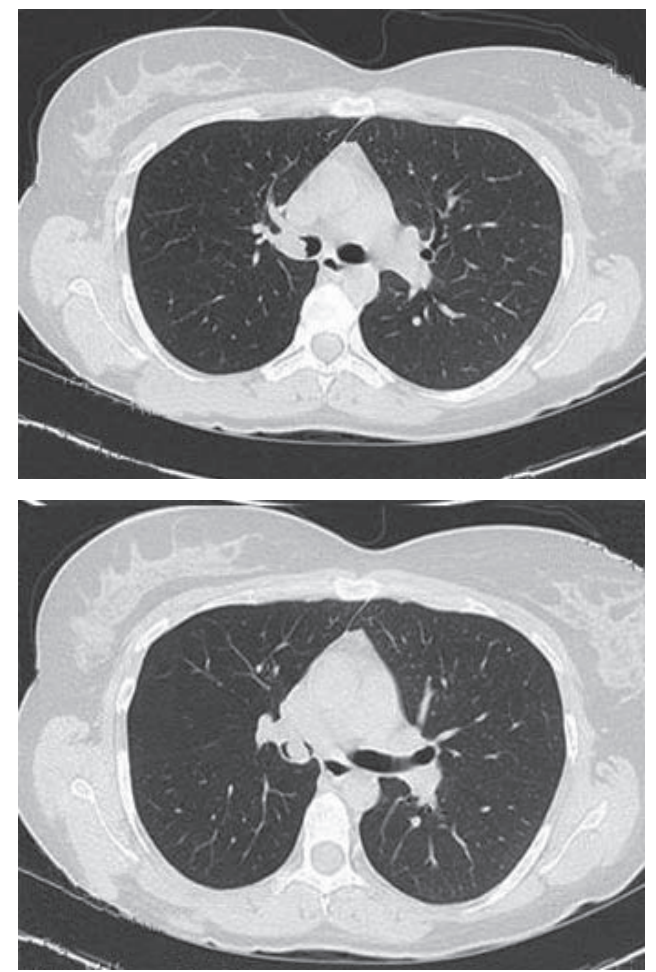

Figure 2

Chest CT section.

\section{Answer 2}

a) Unlikely in a 39-yrold patient who never smoked.

b) Possible with the history of bronchial asthma, positive methacholine bronchoprovocation test and concavity of the expiratory curve.

c) Possible with flattening of the inspiratory and expiratory curve.

d) Unlikely with normal lung volumes.

\section{Answer 3}

Chest CT shows an endobronchial mass originating from the right upper lobe, subtotally obstructing the right main bronchus, but no atelectasis.

Fibre-optic bronchoscopy confirmed a sharply defined tumour which moved with respiration, occluding the main right bronchus (figure 3 ).

Passage around the soft pinkish lesion by the bronchoscope was impossible; the peduncle was fixed in the bronchus of the right upper lobe. Cytology of bronchial washings and needle aspiration showed no malignant cells. No biopsy was taken because of the danger of bleeding in the case of a possible adenoma or carcinoid tumour. We abstained from a resection by rigid bronchosopy because of the large dimension of the tumour, which concealed the diameter of the pedicle. Surgery was necessary for definitive exclusion of malignancy and resection of the peduncle is desired in the case of a carcinoid tumour. Laser or cryotherapy were not considered, in order to avoid destruction of tissue before histological examination. 

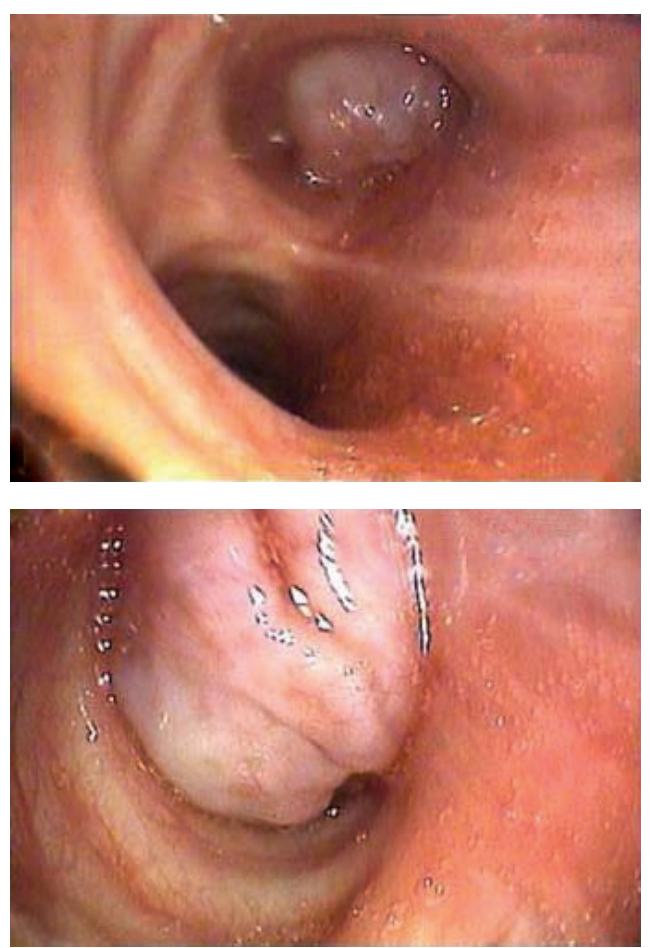

Figure 3

Bronchoscopy with polyp in the right main bronchus.

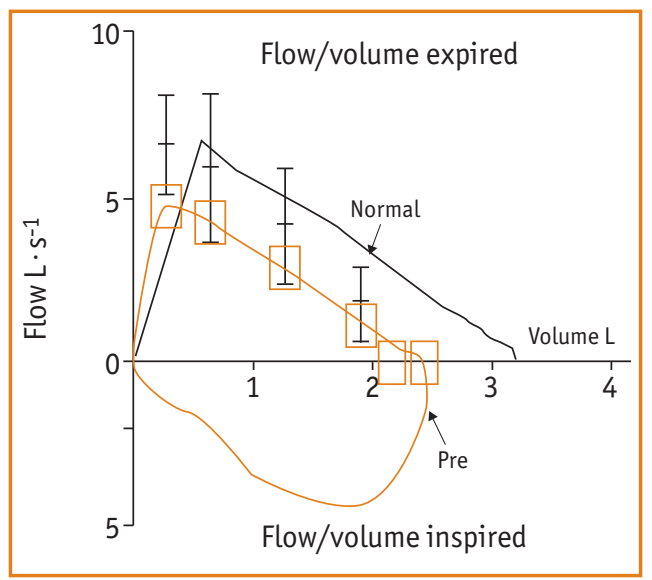

Figure 4

Pulmonary function test after surgery without flattening of the curve.

In order to provide a histological diagnosis, as well as therapy, resection of the right upper lobe was performed. Since the tumour entered the right main bronchus, a sleeve resection of the upper lobe re-adapting the main to the intermediary bronchus was necessary. The frozen section showed no malignancy. Macroscopically, it was a polypoid mass of $45 \times 10 \mathrm{~mm}$. Histopathology revealed a fibroepithelial polyp with no signs of malignancy.

The postoperative course was complicated by severe acute liver failure, probably caused by paracetamol and/or amoxicillin/clavulanic acid.
3 months later, the patient was again seen for a consultation; she was free of symptoms and pulmonary function tests showed a mild restriction explained by the lobectomy, no obstruction and no flattening of the curve (figure 4).

\section{Discussion}

Benign tumours of the tracheobronchial tree are rare ( $<2 \%$ of all lung tumours) [1]. Benign epithelial tumours are classified by their histopathological features. They can be divided into hamartomas, benign fibrous tumours, inflammatory pseudotumours, lipomas, leiomyomas and rare cases; e.g. haemangiomas, adenoma of the mucus glands or mixed tumours. An endobronchial location was observed by Borczuk [2] in only $6 \%$ of these tumours. Very few cases of fibroepithelial polyps have been described in the literature [1-9]. The aetiology of fibroepithelial polyps is unknown. Due to a suspected association to some underlying irritation, they are also called inflammatory polyps. Their fibrovascular core is lined by epithelium [1]. An inflammatory genesis is one of the hypotheses.

IDEURA et al. [3] recently published a case report of a fibroepithelial bronchial polyp in a patient with immunoglobulin M deficiency. The link between these two factors is not clear, but immunoglobulin deficiency with associated repeated airway infections would be compatible with the inflammatory hypothesis [3]. Neither chemical irritation nor infection could provoke bronchial polyps in dogs [4]. Another possible aetiology is a reactive process to a foreign body. However, the localisation in the right upper lobe bronchus in the present patient argues against this foreign body theory, because aspirated particles are most often located in the lower lobes. The proven bronchial hyperreactivity and the respiratory symptoms, such as exercise-induced dyspnoea and cough, are compatible with bronchial asthma and point towards the inflammatory theory in this case.

Fibroepithelial polyps initially cause only slight and nonspecific clinical symptoms. Symptoms are usually cough, (bloody) expectorations and dyspnoea [5]. Chest pain and fever have also been described [4]. A final diagnosis is usually made late in the course of the disease. In this patient, asymmetric auscultation findings and irreversible airway obstruction in spirometry, despite steroid treatment, led to further diagnostic investigations, including $C T$ and bronchoscopy. There are different therapeutic approaches to endobronchial polyps [6]. In the case of an 
inflammatory bronchial polyp, inhaled corticoids led to a regression of the polyp [7]. A precondition for this treatment was a nonobstructing position in large airways. Also, transbronchial polypectomy has been performed. This is not always easy because of the size, bleeding and possible perforation of the airways.

Other therapeutic approaches, such as endoscopic neodymium-doped yttrium aluminium garnet laser, argon plasma coagulation, cryotherapy and photodynamic therapy, have been discussed in the literature, but are rarely used [5].

We recommend complete surgical resection, which allows definitive diagnosis and treatment. Due to the origin of the polyp in the right upper lobe bronchus, a bronchoplasty of the right mainbronchus was not possible in the present patient.

\section{References}

1. Jeong MK, Jung IJ, Seog HP. Benign tumors of the tracheobronchial tree: CT-pathologic correlation. AJR Am J Roentenol 2006; 186: 1304-1313.

2. Borczuk AC. Benign tumors and tumorlike conditions of the lung. Arch Pathol Lab Med 2008; 132: 1133-1148.

3. Ideura G, Agematsu K, Komatsu Y. Selective IgM deficiency accompanied with IgG4 deficiency, dermal complications and a bronchial polyp. Allergol Int 2008; 57: 99-195.

4. Rowlands DT. Fibroepithelial polyps of the bronchus: a case report and review of the literature. Chest 1960; 37 : 199-202.

5. Schnader J, Harrell J, Mathur P, et al. Clinical conference on management dilemmas: bronchiectasis and endobronchial polyps. Chest 2002; 121: 637-643.

6. Amin PB, Baciewicz F. Benign fibroepithelial polyp arising in the bronchus. Arch Surg 2009; 144: 1081-1083.

7. Niimi A, Amitani R, Ikeda T, et al. Inflammatory bronchial polyps associated with asthma: resolution with inhaled corticosteroid. Eur Respir J 1995; 8: 1237-1239.

8. Dinçer I, Demir A, Akin H. A giant endobronchial inflammatory polyp. Ann Thorac Surg 2005; 80: 2353-2356.

9. Barzo P, Malnar L, Minik K. Bronchial papillomas of various origins. Chest 1987; 92: 132-136. 